\title{
Caracterización de eventos adversos perioperatorios en hospitales de nivel III en Medellín-Antioquia
}

\author{
Arias Botero JH. ${ }^{1,2}$, Gómez Arias RD. ${ }^{1}$, González Gaviria P. ${ }^{1}$, Segura Cardona AM. ${ }^{1}$, Acosta Rodríguez F. ${ }^{1}$ \\ 1 Universidad CES, Medellín, Colombia. \\ 2 Sociedad Colombiana de Anestesiología y Reanimación, Bogotá, Colombia.
}

Introducción: Los eventos adversos en salud (EA), entendidos como las complicaciones derivadas del proceso de atención, han cobrado interés dadas las implicaciones a nivel de seguridad, calidad y costos. Aproximadamente 50\% de los EA se relacionan a procedimientos quirúrgicos. Aunque se han hecho estudios a nivel hospitalario, existe poca información de EA en el escenario perioperatorio.

El objetivo del presente estudio fue caracterizar los eventos adversos perioperatorios en 5 instituciones de nivel III en Medellín (Colombia).

Métodos: Se revisaron los registros de todas las atenciones quirúrgicas durante 1 mes en 5 instituciones de nivel III de atención en Medellín (Antioquia), con volúmenes de cirugía mayores a 300 procedimientos/mes. Se tamizaron posibles EA aplicando la herramienta Surgical Trigger Tool.

Se analizó la atención quirúrgica, utilizando el instrumento MRF-2 para confirmar la existencia de EA. Se analizaron las características del paciente, el procedimiento quirúrgico y el evento, evaluando la severidad y la evitabilidad.

Los datos fueron tomados por auditores entrenados, con experiencia en la identificación de EA.

Se presenta frecuencia absoluta y relativa para variables cualitativas y mediana con Riq para variables cuantitativas. Para comparar variables según severidad se utilizó prueba de ?2 y U de Mann Whitney.

Resultados: Se revisaron 3.944 historias clínicas, se detectaron 153 paciente con eventos perioperatorios (Incidencia acumulada 3,88 eventos por 100 procedimientos/mes IC 95\% [3,28 - 4,48].

El $72 \%$ de los EA ocurrieron en pacientes ASA 1 y 2, en su mayoría en pacientes programados, de consulta primaria en la jornada diurna (Figura 1). Se encontró asociación de la severidad del evento con la edad, la especialidad, la urgencia del procedimiento y especialmente con la jornada de trabajo (Figura 2).

El 54\% de los eventos se consideraron evitables.

Conclusiones: En la población estudiada los EA son más frecuentes en pacientes y procedimientos considerados de bajo riesgo acorde a lo reportado en estudios de reclamaciones. La distribución por especialidad y la severidad de los EA también concuerdan con la literatura. Llama la atención el alto porcentaje de eventos considerados evitables.

La incidencia encontrada fue baja al comparar con otros estudios, lo que podría explicarse por la exclusión de las infecciones de sitio operatorio. La jornada de la tarde, la ocurrencia en recuperación y algunas especialidades aumentan el riesgo de eventos severos. Se requieren estudios analíticos que evalúen factores asociados a la presentación de EA.

https://doi.org/10.25237/congresoclasa2019.74 